\title{
A novel technique allowing expedited surgical reconstruction of convex auricular defects without perichondrium
}

\author{
Morgan S. Keefe ${ }^{1}$, Michael A. Keefe ${ }^{* 2}$ and Dillon K. Keefe ${ }^{3}$ \\ ${ }^{1}$ Midwestern University Arizona College of Osteopathic Medicine, Glendale, AZ, USA \\ ${ }^{2}$ Division of Head \& Neck Surgery, Sharp Rees Stealy Medical Group, San Diego, CA, USA \\ ${ }^{3}$ University of Notre Dame, South Bend, IN, USA
}

\section{Clinical Image}

Skin cancer of the ear, most commonly Basal or Squamous Cell cytologic subtypes, is most commonly found on the helical rim, posterior auricle skin and antihelix. Auricular lesions make up nearly $8 \%$ of all skin cancers because of the unique exposure of the auricular structure [1]. Auricular defects pose challenges to reconstruction because not only do the defects vary in location, but more importantly they may involve a combination of skin and cartilaginous tissue (composite defect), which can result in obvious cosmetic deformity. While the concavity and convexity of auricular anatomy add an additional layer of difficulty when it comes to reconstructive surgery, the key concern is the presence or absence of intact perichondrium in the area of defect, particularly on the anterior/lateral helix where there is no subcutaneous tissue providing motility of the skin that would allow for primary defect or flap closure. This leaves the option of secondary intention healing after removal of underlying cartilage (usually using a 3 or $4 \mathrm{~mm}$ punch) to allow for delayed granulation to grow through the punch defects, followed by delayed skin grafting for large convex defects or secondary intention for concave defects in, for example, the conchal bowl or triangular fossa [2-4]. Convex defects, as discussed, require a staged skin graft on an established granulation bed for optimal healing. Other reconstructive/closure options such as the "star" or "wedge" resection of the cartilage will result in a change in the ear height with possible asymmetric appearance of the ears [5]. Also with this technique there is often cartilage buckling with aberrant healing.

Specific to convex defects where the perichondrium has been resected with the tumor while the cartilage remains intact, there have been limited options described for repair. Resection of the underlying cartilage and skin grafting on the tissue bed from the posterior auricular skin allows the repair to be done with one only post-operative visit, but often compromises the aesthetic result due to abnormalities in the ear contour that result from the resection of the cartilage scaffold. A technique to preserve the ear structure involves the use of a 2 or $3 \mathrm{~mm}$ punch to excise regularly spaced cartilage circles of cartilage, which allows granulation tissue to grow through the auricle from the postauricular tissue bed over 7-10 days post-operatively, at which point either a skin graft has then been used to then graft over the granulation tissue or the defect can be allowed to heal via second intention.This regularly-spaced punch technique allows for a better aesthetic appearance, but involves more patient care, a higher risk of infection and repeat visits to the surgeon. Also, while secondary intention healing can be used for convex defects (though it is not ideal), it is a preferable technique for concave or flat surfaces because the scar formed is usually depressed [3].

The technique described here and presented in Figures 1 and 2 offers an alternative approach to repair of larger defects of convex auricular surfaces utilizing grafts without the delay between initial cartilage excision and graft placement. We use a multiple- $3 \mathrm{~mm}$ punch technique, with each punch spaced approximately $3 \mathrm{~mm}$ from surrounding cartilage excision punches (Figure 1). The graft is then placed and secured using through and through 4-0 Nylon suture and Xero form and Telfa compression dressing overtop to provide compression on both sides - anterior and posterior - of the auricle. This technique allows for graft imbibition and survival prior to capillary inosculation at approximately 36 hours post-graft. The dressing is then removed approximately 7-10 days post-operatively with the result as shown (Figure 2). In summary, this technique uses a similar approach to techniques previously used with multiple 3 or $4 \mathrm{~mm}$ punch sites to remove cartilage circles with a corresponding 3-4 mm distance between punch sites. Larger punch sizes are more visible and,

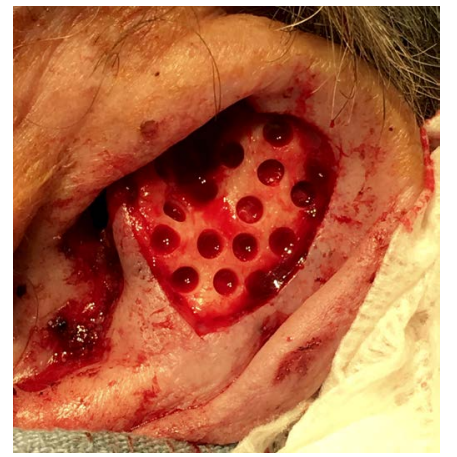

Figure 1. Multiple equidistant $3 \mathrm{~mm}$ cartilage excision punch technique used in auricular antihelix defect reconstruction.

Correspondence to: Michael A. Keefe, Department of Head and Neck Surgery, 2929 Health Center Drive San Diego, CA 92123, USA; Tel: 619-992-7223; 866744-0240; E-mail: Michael.KeefeMD@sharp.com

Received: August 15, 2016; Accepted: August 23, 2016; Published: August 25, 2016 


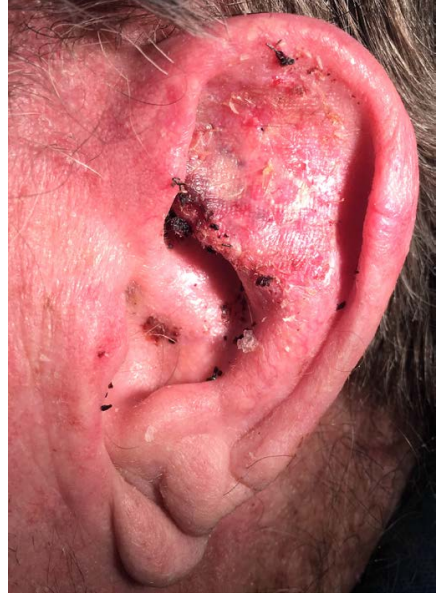

Figure 2. Post-op day 10 bolster removal in auricular antihelix reconstruction using our technique.

while they do not necessarily change the survival, they may alter the structural support. The key advantage in the use of this technique isthe ability for the immediate placement of the graft and use of standard skin graft compression dressings rather than waiting for 7-10 days post-operatively for granulation tissue growth prior to graft placement.

The use of multiple 3 or $4 \mathrm{~mm}$ punches for cartilage excision is advantageous from a cosmetic as well as from a structural standpoint because this technique does not show the cartilage punch defects as much as larger punch circles. This technique certainly is far better cosmetically and structurally than a single excision area would be upon complete healing. In fact, the large single cartilage defect along the antihelix and the associated cosmetic and structural deformity was the motivating factor for the development of the surgical technique we have discussed here.
As noted above, the standard repair for this type of auricular reconstruction has required post-operative time for granulation tissue formation and growth through the cartilage excision sites to develop a bed suitable for skin grafting. Patients were sent home post-operatively with a dressing that requires changing regularly before the second procedure (graft placement) was to take place. At the most basic level, eliminating this delay using the technique we have described is advantageous in that it allows for increased patient comfort by eliminating an extra secondary grafting procedure following the initial excision procedure with the associated two more visits.

In addition to the logistics of trying to coordinate multiple procedures, particularly becausea large portion of these auricular reconstruction patients are elderly patients, this technique also decreases cost associated with multiple visits, improves the physicians' clinic access and, probably most importantly, decreases the risk of problems (such as infection) that can occur with open wound management at home.

\section{References}

1. Leibovitch I, Huilgol SC, Richards S, Paver R, Selva D (2006) The Australian Mohs database: short-term recipient-site complications in full-thickness skin grafts. Dermatol Surg 32: 1364-1368.[Crossref]

2. Wines N, Ryman W, Matulich J, Wines M (2001) Retrospective review of reconstructive methods of conchal bowl defects following mohs micrographic surgery. Dermatol Surg 27: 471-474.[Crossref]

3. Kato H, Watanabe S, Nakamura M, Ijichi K, Morita A (2014) Reconstruction of the external auditory canal using the random flap technique and laser Doppler evaluation. Dermatol Surg. 40:739-742. [Crossref]

4. Park SS, Hood RJ (2001) Auricular reconstruction. Otolaryngol Clin North Am 34 713-738, v-vi.[Crossref]

5. Brent B (1977) The acquired auricular deformity. A systematic approach to its analysis and reconstruction. Plast Reconstr Surg 59: 475-485.[Crossref]

Copyright: (C2016 Keefe MS. This is an open-access article distributed under the terms of the Creative Commons Attribution License, which permits unrestricted use, distribution, and reproduction in any medium, provided the original author and source are credited. 